\title{
Disease and Diagnosis
}

\section{The Role of the Mask in Preventing and Controlling the Coronavirus Disease 2019 Pandemic}

\author{
Abdolhamid Tajvar ${ }^{1,2}{ }^{\circledR}$, Ali Mouseli ${ }^{3}$, Hamid Reza Ghaffari ${ }^{* *}$ \\ ${ }^{1}$ Student Research Committee, Shiraz University of Medical Science, Shiraz, Iran. \\ ${ }^{2}$ Department of Occupational Health and Safety Engineering, Faculty of Health, Hormozgan University of Medical \\ Sciences, Bandar Abbas, Iran. \\ ${ }^{3}$ Social Determinants in Health Promotion Research Center, Research Institute for Health, Hormozgan University of \\ Medical Sciences, Bandar Abbas, Iran. \\ ${ }^{4}$ Department of Environmental Health Engineering, Faculty of Health, Hormozgan University of Medical Sciences, Bandar \\ Abbas, Iran.
}

\begin{abstract}
Following the outbreak of novel coronavirus disease 2019 (COVID-19), there have been debates among scientific communities, officials, and the public about the role of the mask in controlling and preventing this disease. Nonetheless, there are some ambiguities regarding various aspects of the mask which are still important to be addressed, including the type of the mask to be used, the situation in which the mask should be used, the correct way to use the mask, and the effectiveness of the mask in preventing the disease. Accordingly, addressing these ambiguities could change policy trends and public beliefs about the use of mask and help control the disease. To this end, this study discusses the role of the mask in preventing and controlling the COVID-19 pandemic.

Keywords: COVID-19, Cloth mask, N95 respirators
\end{abstract}

Received: June 8, 2020, Accepted: August 26, 2021, ePublished: December 30, 2021

\section{Introduction}

Since the outbreak of coronavirus disease 2019 (COVID-19), the role of the masks in preventing and controlling the prevalence of this disease has become one of the most controversial issues in the world (1). In some countries such as China, people have been forced to wear masks in social settings since the first weeks of the outbreak $(1,2)$. In the newest interim guidance published on 5 June 2020, the World Health Organization (WHO) has updated its advice for the use of the mask and recommended the public to wear masks when going outdoors $(3,4)$.

In addition, there are important considerations about how to use masks, including fit test and seal check for N95 respirators, and handwashing before and after using for all masks. The effectiveness of the mask reduces if these factors are not considered by wearers, leading to an increase in the risk of severe acute respiratory syndrome coronavirus 2 (SARS-CoV-2) transmission $(5,6)$.

Many studies have confirmed that tiny droplets that are thrown out of a patient's mouth or nose during sneezing and coughing can directly or indirectly cause COVID-19 (7). Therefore, if the safe distance of at least 3 feet (one meter) is not considered, the virus can spread between interacting people in close proximity who are not wearing masks while are coughing and sneezing $(4,8)$.

The droplets coming out of the afflicted person's mouth and nose may also settle on inanimate objects, spreading an infection to other people through touching these surfaces, followed by touching their eyes, nose, or mouth (9). Thus, masks can play a pivotal role in reducing the spread of infection to a person through the environment and surrounding surfaces $(10,11)$. Due to the lack of vaccines and specific treatment for COVID-19, it is recommended that decision-makers in health organizations and government agencies review and correct their policies regarding the use of masks by the public as one of the effective strategies for reducing and controlling the prevalence of this virus (12).

In general, respiratory protection equipment (RPE) is classified into two categories depending on their type of efficiency. The first category is respirators, which are designed to protect people against viruses, bacteria, and particulate matters. According to the type of the standard defined for them and their efficiency, they are divided into different names and classes (11). The WHO has recommended the use of the N95 respirator type to protect individuals, especially medical staff from

(C) 2021 The Author(s). This is an open access article distributed under the terms of the Creative Commons Attribution License (http:// creativecommons.org/licenses/by/4.0/), which permits unrestricted use, distribution, and reproduction in any medium, provided the original work is properly cited. 
SARS-CoV-2 (2). The second category of RPE is surgical masks (face masks). These masks are mainly used to prevent the spread of the virus and other infectious agents from a person to the environment, and the WHO has recommended such masks for patients, people suspected to have COVID-19, or those who are taking care of patients at home $(2,11)$.

Taken together, there are some important tips on how to correctly use masks, explaining how failure to pay attention to these crucial tips can increase the risk of people catching SARS-CoV-2. Some of these tips are highlighted as follows:

\section{Tip 1: Do not Use N95 Respirators With Exhalation} Valve

Unfortunately, many people think that the valve installed on N95 respirators acts as a filter while the valve allows exhaled air to escape more quickly and easily, making it more convenient to breathe (12). Hence, when patients or suspected people with COVID-19 infection use such respirators, they inadvertently spread the virus through these valves in the community and at work and family places. Therefore, given the confirmation of the possibility of SARS-CoV-2 transmission through patients with mild symptoms and even asymptomatic cases, and diagnosis difficulty, it is recommended that such people do not use N95 respirators (13).

Tip 2: The Optimal Performance of N95 Respirators Relies on the Success of the Fit Test and Seal Check Due to the fact that N95 respirators are tight-fitting, the wearer needs to do a fit test and seal check before using them in order to check if they work properly. A fit test is conducted to verify that a respirator is both comfortable and correctly fits the wearer's face. In addition, the fit test must be done before users first wear the respirator in order to ensure that the respirator's facepiece fits their face, and must also be re-tested at least every 12 months (14).

Furthermore, the seal check is a quick check that must be performed by the wearer of the N95 respirator each time it is put on. If the respirators leaks, microdroplets, and aerosols including coronavirus will be pulled into the facepiece and can be breathed in. Therefore, the wearer must ensure that there are no obstacles or gaps such as beards, mustaches, moles, or pimples between the edges of the mask and the face (15).

Nevertheless, these types of respirators (i.e., N95 respirators without an exhalation valve) are more suitable for the medical care staff because some of the medical operations are aerosol-generating procedures, and there is a possibility of transmitting the coronavirus to them through patients' secretions. For the public, it will be sufficient to wear only face masks $(2,16)$.
Tip 3: Need to Use Masks in a Closed-roof Work Places Patients with mild symptoms and even asymptomatic ones can be considered as a source of infection and directly or indirectly transmit the virus to their co-workers in the surrounding area. Given that the detection of such people is very difficult, it is recommended that all employees who are working in the same room or a roofed environment must wear a surgical mask (1).

Tip 4: If You Do Not Have Access to Surgical Masks or Standard N95 Respirators, You Can Use Cloth Masks Considering Hygienic Protocols

As discussed above, if a person does not have access to surgical masks or N95 respirators, in the current crisis, the US Center for Disease Control and Prevention (CDC) has recommended using cloth face coverings by the general public as health precautions $(9,17)$. This will prevent the virus from spreading during coughing, sneezing, or talking, and thus protect the person against other people's secretions (12). Moreover, given that people subconsciously touch their mouth, nose, and eyes (i.e., the T-zone), wearing a mask prevents the transmission of possible viruses on the hand to the T-zone.

Tip 5: The Effectiveness of Masks Depends on the Hygiene of the Hands

The WHO has mentioned that the use of masks is effective when accompanied with the principles of hand hygiene and frequent washing with soap and water or disinfection with alcohol solutions before using and before and after removing the mask. Otherwise, by touching the outer body of the mask, there is a possibility of virus transmission (2).

\section{Conclusion}

According to the recommendation of the WHO, if there is widespread community transmission, especially in settings where physical distancing cannot be maintained, governments should encourage the general public to wear masks (4). Therefore, it is critical for public health agencies and governments to make rational and practical advice and develop universal guidelines on appropriate mask use to effectively curb this pandemic which is already wreaking havoc on communities and families welfare.

Conflict of Interest Disclosures

The authors declare that there is no conflict of interests.

Acknowledgments

Not applicable.

Ethical Statement

All authors have been personally and actively involved in substantive work leading to the manuscript and will hold themselves jointly and individually responsible for its content. 


\section{Authors' Contribution}

AT ,AM and HRG: Giving ideas and drafting and revising the article.

\section{Funding/Support}

There is no funding support.

\section{Informed Consent}

Not applicable.

\section{References}

1. Feng S, Shen C, Xia N, Song W, Fan M, Cowling BJ. Rational use of face masks in the COVID-19 pandemic. Lancet Respir Med. 2020;8(5):434-6. doi: 10.1016/s2213-2600(20)30134-x.

2. World Health Organization (WHO). Advice on the Use of Masks in the Community, During Home Care and in Healthcare Settings in the Context of the Novel Coronavirus (2019-nCoV)|'Outbreak: Interim Guidance, 29 January 2020. WHO; 2020.

3. World Health Organization (WHO). Advice on the Use of Masks in the Context Of COVID-19: Interim Guidance, 5 June 2020. WHO; 2020.

4. World Health Organization (WHO). Coronavirus Disease (COVID-19) Advice for the Public. WHO; 2020. https:// www.who.int/emergencies/diseases/novel-coronavirus-2019/ advice-for-public.

5. Gamage B, Moore D, Copes R, Yassi A, Bryce E. Protecting health care workers from SARS and other respiratory pathogens: a review of the infection control literature. Am J Infect Control. 2005;33(2):114-21. doi: 10.1016/j. ajic.2004.12.002

6. Occupational Safety and Health Administration (OSHA). Respiratory Protection. OSHA; 2002.

7. Leung CC, Lam TH, Cheng KK. Mass masking in the COVID-19 epidemic: people need guidance. Lancet. 2020;395(10228):945. doi: 10.1016/s0140-6736(20)305201.

8. Lai CC, Shih TP, Ko WC, Tang HJ, Hsueh PR. Severe acute respiratory syndrome coronavirus 2 (SARS-CoV-2) and coronavirus disease-2019 (COVID-19): the epidemic and the challenges. Int J Antimicrob Agents. 2020;55(3):105924. doi: 10.1016/j.ijantimicag.2020.105924.

9. Kampf G, Todt D, Pfaender S, Steinmann E. Persistence of coronaviruses on inanimate surfaces and their inactivation with biocidal agents. J Hosp Infect. 2020;104(3):246-51. doi: 10.1016/j.jhin.2020.01.022.

10. MacIntyre CR, Chughtai AA. Facemasks for the prevention of infection in healthcare and community settings. BMJ. 2015;350:h694. doi: 10.1136/bmj.h694.

11. Liverman CT, Larson EL. Preventing Transmission of Pandemic Influenza and Other Viral Respiratory Diseases: Personal Protective Equipment for Healthcare Personnel: Update 2010. Washington, DC: National Academies Press; 2011.

12. Worby $\mathrm{CJ}$, Chang $\mathrm{HH}$. Face mask use in the general population and optimal resource allocation during the COVID-19 pandemic. medRxiv. 2020. doi: 10.1101/2020.04.04.20052696.

13. Hoehl S, Rabenau H, Berger A, Kortenbusch M, Cinatl J, Bojkova D, et al. Evidence of SARS-CoV-2 infection in returning travelers from Wuhan, China. $N$ Engl J Med. 2020;382(13):1278-80. doi: 10.1056/NEJMc2001899.

14. Occupational Safety and Health Administration (OSHA). Respiratory Protection. Final rule. 1998. Available from: https://www.osha.gov/laws-regs/federalregister/1998-01-08.

15. Roberge RJ. Evaluation of the rationale for concurrent use of N95 filtering facepiece respirators with loose-fitting powered air-purifying respirators during aerosol-generating medical procedures. Am J Infect Control. 2008;36(2):135-41. doi: 10.1016/j.ajic.2007.04.284.

16. Danyluk Q, Hon CY, Neudorf M, Yassi A, Bryce E, Janssen $B$, et al. Health care workers and respiratory protection: is the user seal check a surrogate for respirator fittesting? J Occup Environ Hyg. 2011;8(5):267-70. doi: 10.1080/15459624.2011.566016.

17. Kampf G. Potential role of inanimate surfaces for the spread of coronaviruses and their inactivation with disinfectant agents Infect Prev Pract. 2020;2(2):100044. doi: 10.1016/j. infpip.2020.100044. 